\title{
Diversité génétique, variation géographique et flux géniques chez quelques lépidoptères rhopalocères français
}

\author{
Henri Descimon, Marie Zimmermann, Emmanuel Cosson, \\ Bernard BARAscud, Gabriel NÈve
}

Laboratoire de systématique évolutive, Case 5, Université de Provence, 3 place Victor Hugo, F-13331 Marseille cedex 3, France

\begin{abstract}
Résumé - Nous présentons une étude synthétique et comparative de la structure génétique des populations de trois papillons diurnes français, Proclossiana eunomia, Parnassius apollo et Euphydryas aurinia. Grâce à l'électrophorèse des allozymes, il a été possible d'estimer comparativement le niveau de variation génétique à l'intérieur des divers peuplements des espèces étudiées. Ces données permettent également de proposer des éléments d'explication de cette variation : fonctionnement actuel des populations et métapopulations, histoire des biomes ouest-européens au cours des vingt derniers millénaires. Chacun des cas étudiés possède une spécificité biogéographique : distribution de type « relique glaciaire » très disjointe $(P$. eunomia $)$, espèce de montagne largement répandue, mais en forte régression $(P$. apollo $)$, espèce très diversifiée écologiquement $(E$. aurinia). D'une manière générale, les populations méridionales montrent des variations importantes au niveau local et régional et présentent une structuration géographique nette. Les populations septentrionales montrent une diversité génétique plus faible et une structuration spatiale confuse, avec un effet d'isolement par la distance faible ou absent. Ce phénomène est le plus accusé pour les populations du Nord-Est du Massif Central de $P$. eunomia, qui résultent d'une introduction délibérée datant de 25 ans. Dans le cas d'E. aurinia, l'adaptation à des plantes nourricières différentes est aussi liée à la différenciation génétique et à sa variation géographique. Ces caractéristiques peuvent être au moins partiellement expliquées par la colonisation postglaciaire du nord de l'Europe à partir des refuges méridionaux où les biomes tempérés et méditerranéens étaient concentrés pendant les glaciations. Cette colonisation s'est accompagnée d'une perte de diversité génétique et d'une variation sans structuration géographique nette. Par ailleurs, la diversification écologique très élevée du sud de l'Europe permet et a permis une plus grande diversification génétique. Le glissement vers le nord des aires de nombreuses espèces risque donc de s'accompagner d'une perte de diversité génétique.
\end{abstract}

diversité génétique / flux géniques / variation géographique / refuges / lépidoptères

* Correspondance et tirés-à-part

E-mail: lsysevol@newsup.univ-mrs.fr 


\begin{abstract}
Genetic variation, geographic variation and gene flow in some French butterfly species. We present here a comparative and synthetic study of the genetic structure of the populations of three French butterfly species, Proclossiana eunomia, Parnassius apollo and Euphydryas aurinia. Using allozyme electrophoresis, it is possible to estimate the level of genetic variation within and between populations of these species in various regions of France and neighbouring countries. These data also allow us to suggest an explanation of this variation: present functioning of populations and metapopulations, adaptation to foodplants and history of the European biomes during the last twenty millenia. Each of the presented cases presents a biogeographical specificity: very disjunct distribution of the "glacial relict" type (Proclossiana eunomia), widespread mountain species, presently in strong regression (Parnassius apollo), ecologically very diversified species (Euphydryas aurinia). Generally speaking, genetic variation is large at both local and regional scale in southern regions where the genetic structure follows a clear geographic structure. Northern populations show a lower genetic diversity and a confused genetic structure, with an isolation-by-distance effect lower or absent. This phenomenon is the most obvious in $P$. eunomia populations resulting from a deliberate introduction in northeastern Massif Central 25 years ago. In the case of E. aurinia, the adaptation to different foodplants plays a rôle in genetic differentiation and its geographic variation. These characteristics can be explained at least in part by the postglacial colonization of northern Europe from southern refugia where temperate and mediterranean biomes were concentrated during glacial times. This extension has been accompanied by a loss of genetic diversity and a variation without clearcut geographic organization. Otherwise, the very large ecological diversity of southern Europe has allowed and still allows a larger genetic differentiation within species. The northwards shift of species distribution associated with present climate change is therefore likely to cause a severe loss of genetic diversity within species.
\end{abstract}

genetic diversity / gene flow / geographic variation / refugia / butterflies

\title{
1. INTRODUCTION
}

Tout organisme peut être un objet d'étude mais, en dernière analyse, l'intérêt porté à un groupe vivant sur le plan scientifique est lié à ce qu'il peut apporter à la société humaine, à la 《ressource » qu'il représente pour notre espèce. Comme les oiseaux ou les orchidées, par exemple, les papillons sont l'objet de l'intérêt d'un grand nombre de naturalistes professionnels et amateurs, produisant une masse de publications dans des revues de niveaux très variés. De riches informations sont donc disponibles, au moins à un niveau naturaliste. Par ailleurs, les lépidoptères sont des phytophages oligophages et l'étude de leurs relations avec leurs plantes nourricières est un chapitre important des études contemporaines en écologie [45]. Ces seuls faits justifient l'assertion de Ford [20], qui souligne l'intérêt des lépidoptères en tant que matériel pour la théorie de l'évolution et donc la connaissance en général. Mais, pour leur donner un statut de ressource complet, il faut au moins qu'ils soient utiles à 
la gestion des territoires, des écosystèmes et de leurs constituants. Le présent exposé tentera de montrer qu'il peut en être ainsi, au moins dans certains domaines.

À l'intérieur des espèces, l'étude des échanges géniques fait appel à des modèles finalement assez simples, et dont la pertinence peut être discutée $[4,54]$. Grâce aux travaux d'Ehrlich [17,18], des lépidoptères, les Mélitées, ont joué un rôle important dans la définition de la structure des populations vis-à-vis des échanges géniques (populations ouvertes ou fermées). Plus récemment, c'est encore chez des Mélitées, européennes cette fois-ci, que Hanski et ses collaborateurs ont développé le modèle des métapopulations créé par Levins [24-26,33]. Les lépidoptères européens ont été l'objet de nombreux travaux de ce type et nous verrons ce qu'ils peuvent apporter à la théorie des échanges géniques.

La biogéographie de l'Europe occidentale montre, tant au niveau de la diversité génétique interspécifique qu'à celui de la diversité génétique intraspécifique, que celles-ci sont inégalement répandues selon les territoires. Il y a généralement coïncidence entre les deux niveaux. Des analyses récentes, portant sur des groupes animaux et végétaux variés [28] ont montré que les variations du climat au cours du Quaternaire, et en particulier les glaciations, ont bouleversé les distributions de la plupart des espèces. Quels sont les déterminants de cette distribution de la biodiversité chez les lépidoptères ? Et, enfin, quelles peuvent être les conséquences des changements climatiques que nos pays sont en train de subir sur cette distribution?

\section{MATÉRIEL ET MÉTHODES}

Comme il s'agit ici d'un travail de synthèse sur des travaux effectués dans notre laboratoire depuis quelques années, nous n'envisagerons pas en détail les méthodes d'acquisition des données ; celles-ci résultent essentiellement de l'analyse du polymorphisme enzymatique par électrophorèse sur acétate de cellulose [57] adaptée aux espèces étudiées $[1,2,40]$. Dans le cas précis des lépidoptères, les enzymes sont en effet à l'heure actuelle les seuls marqueurs nucléaires utilisables permettant de définir les génotypes. L'ADN microsatellite semble être peu abondant ou d'un accès difficile dans les cas étudiés [36,38] et les autres marqueurs ADN actuellement accessibles (RAPDs et AFLPs) sont dominants, ce qui interdit les analyses génotypiques. L'ADN mitochondrial fournit un outil accessible mais modérément variable (même au niveau de la région de contrôle) et là encore d'application limitée.

Le tableau I récapitule les 14 loci trouvés polymorphes et lisibles chez les 3 espèces étudiées, ainsi que le nombre d'allèles qui y ont été détectés. L'analyse des résultats a été effectuée à l'aide des logiciels Biosys1 de Swofford et Selander [49], Genepop de Raymond et Rousset [44]. 
Tableau I. Loci enzymatiques étudiés chez les 3 espèces de lépidoptères diurnes objets de l'article. Pour la signification des abréviations, on se référera à Barascud et al., 1999. Les chiffres correspondent au nombre d'allèles détectés à chaque locus et dans l'ensemble de l'échantillon de chaque espèce. Un tiret (-) correspond à des électrophorégrammes non interprétables avec la méthode utilisée.

\begin{tabular}{|c|c|c|c|c|c|c|c|c|c|c|c|c|c|c|c|}
\hline & & 6PGD & AAT1 & AAT2 & aGPDH & AK & G6PDH & HBDH & IDH1 & IDH2 & MDH & MOD & MPI & PGI & PGM \\
\hline Euphydryas aurinia & France & 1 & 6 & 10 & 6 & 5 & 3 & 8 & 10 & 10 & 1 & 1 & 1 & 16 & 9 \\
\hline Parnassius apollo & France et Espagne & - & 6 & 5 & - & - & 6 & 6 & - & - & 1 & 1 & 7 & 7 & 6 \\
\hline \multirow[t]{2}{*}{ Proclossiana eunomia } & France, Belgique, Espagne & 3 & 3 & 1 & 1 & 2 & 3 & 2 & 1 & 1 & 1 & 1 & 2 & 5 & 2 \\
\hline & Ensemble de l'Europe & 4 & 4 & 2 & 2 & 3 & 3 & 2 & 1 & 1 & 1 & 1 & 7 & 8 & 6 \\
\hline
\end{tabular}


Nous nous limiterons systématiquement aux procédures d'analyse suivantes :

- Dendrogrammes : la combinaison distance de Cavalli-Sforza et Edwards + agrégation par la méthode du « neighbour joining » a été choisie comme fournissant généralement la meilleure image des relations entre les populations [29].

- Projection en espaces réduits : complémentaire de la précédente dans la représentation des relations génétiques entre populations [15,23], elle se limitera ici au plan 1-2 d'analyses factorielles des correspondances où les 《 individus » sont les populations et où la fréquence de chaque allèle est considérée comme une variable.

- Isolement par la distance (IPD) : cette étude passe d'abord par le calcul de la $F_{S T}$ selon la méthode de Weir et Cockerham [52], tenant compte des situations multialléliques (paramètre $\theta$ ) ; le « nombre absolu de migrants », produit de l'effectif (efficace) des populations, $N_{e}$, et du taux de migration, $m,\left(N_{e} m\right)$ est calculé par la formule : $N_{e} m=(1-\theta) / 4 \theta[54,55]$. On procède ensuite à l'analyse de l'autocorrélation [48] entre le produit $N_{e} m$ et le logarithme de la distance chez toutes les paires possibles de l'ensemble des populations étudiées. Un coefficient de corrélation de 0 indique une absence de structuration et un coefficient de -1 (nombre de migrants décroissant régulièrement avec la distance) une structure intégralement déterminée par la position spatiale. Il a été montré qu'en raison des «pseudoréplications » entre paires de populations voisines, le test statistique le plus approprié à évaluer la significativité du coefficient est le test de Mantel [31].

D'un point de vue heuristique, ces méthodes de représentation et d'analyse des structures génétiques feront l'objet d'une comparaison.

\section{3. ÉTUDE DE CAS CHEZ LES LÉPIDOPTĖRES FRANÇAIS ET OUEST EUROPÉENS}

Nous disposons de données sur les populations de plusieurs espèces présentant des cas diversifiés de structures de peuplement, depuis une distribution extrêmement fragmentée à grande échelle jusqu'à une distribution continue en métapopulation. Nous donnerons ici un aperçu des enseignements de l'étude de leur structure génétique dans notre laboratoire, en tenant compte de travaux sur d'autres espèces effectués par ailleurs.

\subsection{Proclossiana eunomia, une espèce à distribution hyper-disjointe}

Cette espèce holarctique possède une distribution circumpolaire continue (à grande échelle) dans la zone froide de la forêt boréale. En Europe de l'ouest, sa distribution est très fragmentée et présente les caractères d'une « relique 
glaciaire ». Elle y montre deux types de colonies, soit très méridionales et montagnardes : aux deux extrémités de la chaîne pyrénéo-cantabrique, dans le sud des Alpes italiennes et autrichiennes et en Bulgarie ; soit, dans le nord de l'Europe, en un «semis » dispersé, souvent hébergé par de très basses montagnes comme l'Ardenne, par exemple. Elle est liée dans nos régions à une seule plante nourricière, la Bistorte (Polygonum bistorta), largement répandue dans les montagnes ouest européennes ; cette Polygonacée est néanmoins absente de la chaîne axiale des Pyrénées.

Nous disposons de données sur la structure génétique des populations des Pyrénées orientales [1], de Bulgarie [37] et d'Ardenne [42]. Par ailleurs, l'espèce a été introduite par l'auteur senior du présent article dans le Morvan dans les années 1970 et les conséquences écologiques et génétiques du processus de colonisation qui en ont découlé ont pu être mises en évidence $[2,40]$.

\subsubsection{La structure génétique d'une métapopulation introduite : les colonies de P. eunomia dans le Morvan}

Ce massif montagneux de basse altitude possède des colonies importantes de Bistorte, plante nourricière de $P$. eunomia ; mais ce papillon n'y était pas connu avant les années 70 . En juin 1970, 4 femelles originaires de l'Ardenne furent relâchées, dans le nord du massif ; en juin 1974, 16 femelles furent implantées, cette fois-ci dans le sud. À partir de ces fondatrices, tout le massif fut progressivement colonisé en une vingtaine d'années $[2,39,40]$. Des populations réparties dans tout le massif ont été étudiées au niveau des allozymes et de la patterne alaire $[2,39]$ et nous résumons ici les conclusions de ce travail. Les conséquences attendues du processus de colonisation ont effectivement été observées : d'une part une dérive notable (et une perte de polymorphisme) au fur et à mesure que l'on s'éloigne des points d'implantation et, d'autre part, une absence de structure organisée ; la projection des populations par Analyse Factorielle des Correspondances à partir des fréquences alléliques révèle une topologie confuse et sans rapport avec la localisation topographique. Il n'y a pas d'effet d'isolement par la distance, comme le montre l'absence de corrélation significative entre distance et $\theta$. On note le comportement aberrant du locus 6PGD : d'une part, il n'est resté polymorphe qu'à proximité de la localité d'implantation où l'effectif était le plus grand ; d'autre part, il présente un déséquilibre de liaison important par rapport aux autres loci. Nous avons pensé qu'il était lié au sexe, mais il est aussi possible qu'il soit lié à un locus soumis à une sélection importante et donc, par « hitch hiking » à un balayage sélectif. D'une manière générale, la structure observée correspond précisément aux premiers stades de l'évolution d'un ensemble de populations colonisatrices, telle qu'elle est prévue par le modèle de Slatkin : les effets stochastiques liés à une dérive importante et à la fondation par une très petit nombre d'individus sont prédominants. 
Diversité génétique chez quelques lépidoptères

(a)

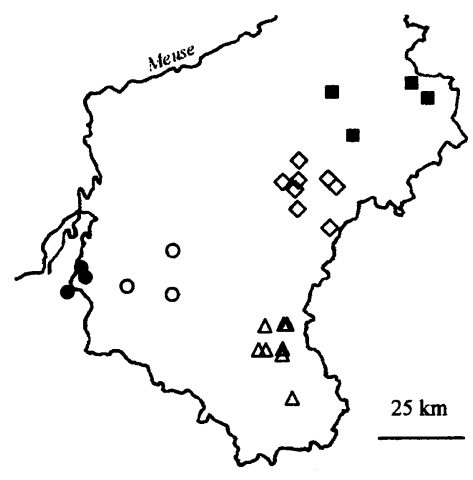

(b)

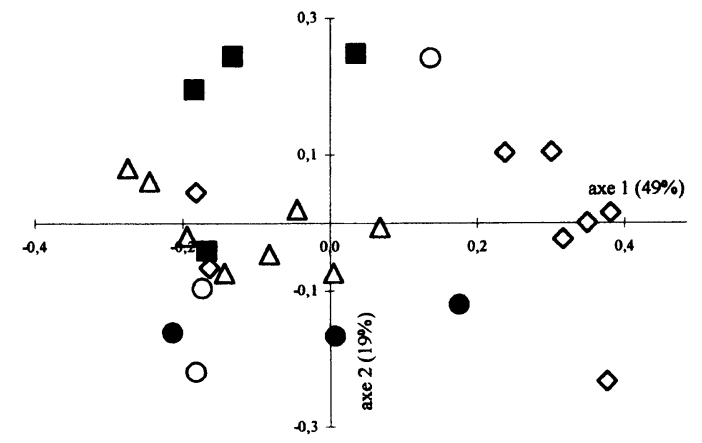

(c)

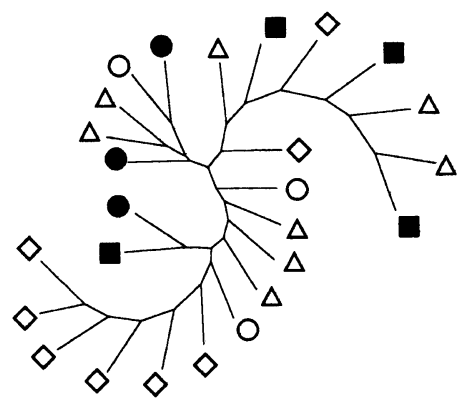

Figure 1. A. Position géographique de 26 populations de $P$. eunomia étudiées dans l'Ardenne ; on a subdivisé cet ensemble en cinq régions représentées chacune par des figurés différents repris en $\mathrm{B}$ et $\mathrm{C}(\boldsymbol{\square}$ : Hautes Fagnes ; $\diamond:$ Tailles ; $\triangle:$ Gaume ; $\circ:$ Ardenne centrale ; $\bullet$ : Ardenne française). B. Position de ces mêmes populations sur le plan 1-2 d'une Analyse Factorielle des Correspondances sur les fréquences alléliques aux loci enzymatiques analysés dans ces populations ( $c f$. Tab. I) ; pourcentage d'inertie représenté par le plan 1-2: $68 \%$; pourcentage de l'inertie totale représenté par chacun des axes (68\% au total). C. Phénogramme obtenu à partir des mêmes données par la distance de Cavalli-Sforza et Edwards et l'agrégation du « neighbour joining $»$. 
(a)

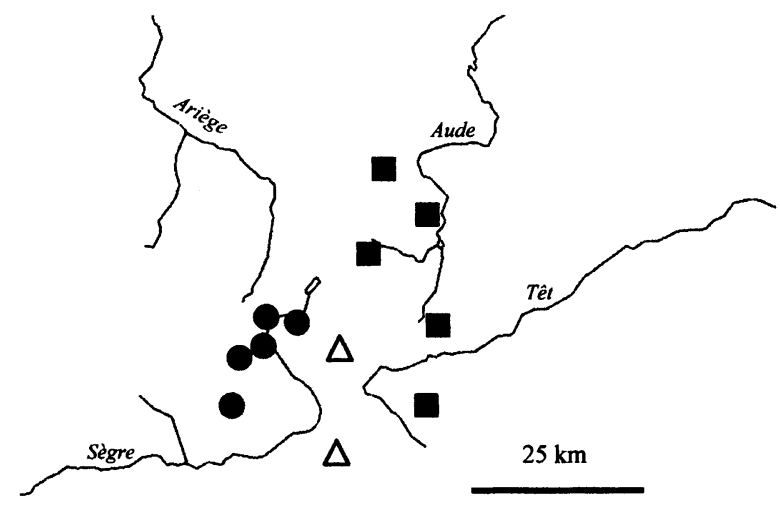

(b)

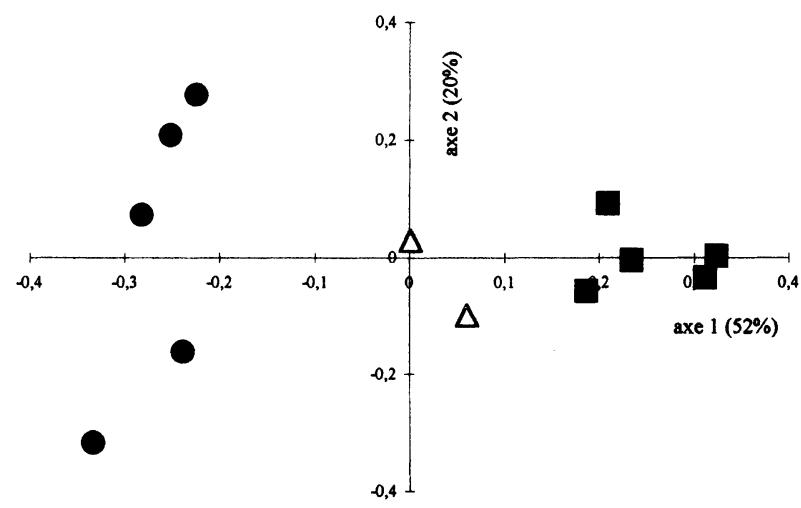

(c)

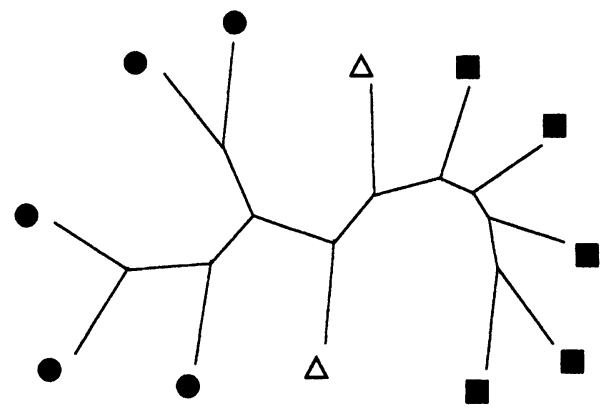

Figure 2. A. Position géographique de 12 populations de $P$. eunomia étudiées dans les Pyrénées orientales ; on a subdivisé cet ensemble en 3 régions représentées chacune par des figurés différents repris en $\mathrm{B}$ et $\mathrm{C}(\bullet$ : Ouest de la région, $\triangle$ : centre, $\mathbf{\square}$ : Est). B. AFC réalisée sur les fréquences alléliques aux loci analysés ; pourcentage d'inertie représenté par le plan 1-2:72\%. C. Phénogramme obtenu avec ces données et par la même méthode qu'en $1 \mathrm{C}$. 
Les résultats d'une analyse multivariée de la patterne alaire sont également intéressants : une structure hiérarchisée géographiquement et un effet d'isolement par la distance s'y révèlent nettement. Nous avons évidemment affaire ici à un ensemble contrôlé par un nombre important de loci, ce qui peut expliquer la netteté plus grande des résultats. Mais on peut penser aussi à une différenciation sous l'effet de la sélection : la patterne alaire possède des fonctions adaptatives bien connues [11] et le Morvan n'a pas un climat homogène du nord au sud. Les populations introduites ont d'ailleurs rapidement divergé de leur population mère ardennaise $[2,39]$.

\subsubsection{Les peuplements de P. eunomia dans l'Ardenne et les Pyrénées orientales}

La hiérarchisation très floue des populations de l'ensemble de l'Ardenne, où $P$. eunomia est distribuée largement mais d'une manière fragmentée, rappelle étonnamment la structure précédente [42]. La représentation par l'AFC ou par des phénogrammes (Fig. 1B, C) montre un découplage marqué entre position géographique et structure génétique. Étudié par la méthode de Slatkin [46], l'effet d'isolement par la distance (Fig. 6A) montre une corrélation très basse quoique significative. En somme, les phénomènes prédominants sont la dérive et l'effet des fondateurs, ce qui s'accorde bien avec les observations écologiques [41].

Les peuplements des Pyrénées Orientales montrent une structure radicalement différente, clairement organisée et hiérarchisée selon les bassins hydrographiques [1]. Cette structure est aussi perceptible sur les phénogrammes que sur l'AFC basée sur les fréquences alléliques ; celle-ci localise les populations sur le plan 1-2 selon une topologie similaire à leur localisation géographique (Fig. 2B, C). Une très nette corrélation entre taux de migration et distance géographique est observée (Fig. 6B).

Par ailleurs, le pool génique de l'ensemble méridional est beaucoup plus riche et diversifié que celui des populations ardennaises ; il y a non seulement plus de loci polymorphes, mais aussi plus d'allèles par locus dans une région donnée et des allèles caractéristiques d'une région donnée (Tab. II). De plus, tous les allèles observés en Ardenne (à part un, d'ailleurs décelé en Bulgarie [37]) sont présents dans les Pyrénées-Orientales.

En tenant compte des enseignements de la colonisation artificielle du Morvan par l'espèce, il est tentant de tracer un scénario de l'histoire du peuplement de $P$. eunomia en Europe occidentale. Pendant la glaciation du Würm, comme les autres espèces de milieux tempérés froids, $P$. eunomia s'est réfugiée bien au sud, et en particulier au pied sud des Pyrénées et des Asturies. À la fin de la glaciation, les conditions climatiques favorables à l'espèce ont « glissé 》 de deux manières : d'une part, vers des altitudes plus élevées et, d'autre part, vers le nord. Les colonies pyrénéo-cantabriques ont donc simplement migré plus haut en montagne et sur de courtes distances ; elles ont conservé l'essentiel 
Tableau II. Estimateurs de la diversité génétique dans les populations de diverses régions des trois espèces étudiées. L'hétérozygotie $\mathrm{H}$ est la moyenne des hétérozygoties calculées locus par locus. Le nombre moyen d'allèles efficaces est calculé à partir de l'hétérozygotie par la formule $N e=1 /(1-H)$. $N_{e} m$ est déduit de $\theta$ par la formule : $N_{e} m=(1-\theta) / 4 \theta$.

\begin{tabular}{|c|c|c|c|c|c|c|c|}
\hline & $\begin{array}{c}\text { Nb de pop. } \\
\text { échantillonnées }\end{array}$ & $\begin{array}{c}\text { \% de loci } \\
\text { polymorphes }\end{array}$ & $\begin{array}{c}\text { Nb moyen d'allèles } \\
\text { par locus }\end{array}$ & $\mathbf{H}$ & $\begin{array}{c}\text { Nb moyen d'allèles } \\
\text { efficaces }\end{array}$ & $\theta$ & $N_{e} m$ \\
\hline \multicolumn{8}{|c|}{ Proclossianna eunomia } \\
\hline Ardenne & 26 & 40 & 1,5 & 0,265 & 1,361 & 0,123 & 1,783 \\
\hline Pyrénées & 12 & 70 & 2 & 0,136 & 1,157 & 0,099 & 2,275 \\
\hline \multicolumn{8}{|l|}{ Parnassius apollo } \\
\hline Alpes & 17 & 100 & 5,71 & 0,3187 & 1,468 & 0,0548 & 4,312 \\
\hline Alpes maritimes & 10 & 100 & 5 & 0,3138 & 1,457 & 0,041 & 5,848 \\
\hline Jura & 2 & 85,70 & 3 & 0,2663 & 1,363 & 0,1244 & 1,760 \\
\hline Pyrénées & 7 & 100 & 4,71 & 0,3715 & 1,591 & 0,0872 & 2,617 \\
\hline Massif Central & 3 & 71,40 & 2,86 & 0,2832 & 1,395 & 0,2658 & 0,691 \\
\hline Espagne & 2 & 85,70 & 2,71 & 0,3134 & 1,456 & 0,1524 & 1,390 \\
\hline \multicolumn{8}{|l|}{ Euphydryas aurinia } \\
\hline Sud de la France & 35 & 100 & 7,2 & 0,2484 & 1,330 & 0,1125 & 1,972 \\
\hline Nord de la France & 26 & 90 & 5,6 & 0,1715 & 1,207 & 0,0648 & 3,609 \\
\hline
\end{tabular}


de leur diversité génétique. Les colonisateurs qui ont migré vers le nord ont effectué une très grande série de sauts, perdant par étapes une partie de leur polymorphisme ; de plus, il n'est pas à exclure qu'aient eu lieu des phases de balayage sélectif. Les colonies intermédaires qui ont occupé un temps le Massif Central (et le Morvan) ont disparu avec le réchauffement (spécialement à l'époque xérothermique) et l'envahissement des espaces ouverts par la forêt (cas du Morvan), en particulier.

\subsection{Parnassius apollo, une espèce à distribution fragmentée}

L'Apollon était naguère présent dans tous les massifs montagneux français d'altitude supérieure à $1000 \mathrm{~m}$ [12]. C'est une espèce spectaculaire et sa variation géographique a été l'objet de nombreuses descriptions de sous-espèces, appuyées sur la seule patterne alaire et effectuées malheureusement selon des méthodes peu rigoureuses. Leur valeur est au mieux indicative. Cependant, le déterminisme génétique de ces variations est souvent assez simple et peut être étudié par croisement ; l'action de gènes majeurs y subit des interactions épistatiques qui peuvent être disséquées par des croisements auxquels cette espèce se prête bien [13].

L'optimum altitudinal de l'espèce se situe entre 1000 et $2000 \mathrm{~m}$, avec des colonies jusqu'à moins de $500 \mathrm{~m}$ et d'autres jusqu'à $2500 \mathrm{~m}$. Dans les Alpes et les Pyrénées, la distribution de l'Apollon est continue ; dans le Jura, jusqu'à une époque récente (fin des années 1960), un réseau de populations assez bien connectées existait du nord au sud. Dans le Massif Central, on distingue deux ensembles assez nettement séparés. Le premier du côté sud-est, allant du Vivarais aux Causses, est nettement fragmenté en petites colonies séparées par des espaces inhospitaliers. Le second, occidental, va du Cantal au Puy de Dôme et il est plus continu. Très restreintes, les colonies vosgiennes se sont éteintes dans les années 1970. Dans les régions à peuplement discontinu, la structure du peuplement est clairement en métapopulation classique [24-26], avec néanmoins des 《 sources » et «puits » bien caractérisés [9] et une dynamique instable. Lié à des milieux très ouverts, l'Apollon se nourrit de Crassulacées et il a besoin de peuplements suffisants de ces plantes pour maintenir des colonies [10].

La structure génétique des populations françaises (auxquelles ont été ajoutées deux populations du centre-ouest de l'Espagne), révélée par une étude encore limitée des allozymes [6], est au premier abord confuse. Sa représentation, par des dendrogrammes comme par l'AFC, montre une correspondance brouillée avec la localisation géographique (Fig. 3B, C). On attendrait évidemment une hiérarchisation en grands massifs, eux-mêmes subdivisés en massifs secondaires, mais ce n'est pas ce qui est observé. Il est possible d'établir un certain parallélisme avec ce qui est observé chez $P$. eunomia, mais à une échelle différente ; certaines populations voisines sont montrées proches génétiquement mais il y a des positionnements étonnants (une population ariégeoise, par 
(a)

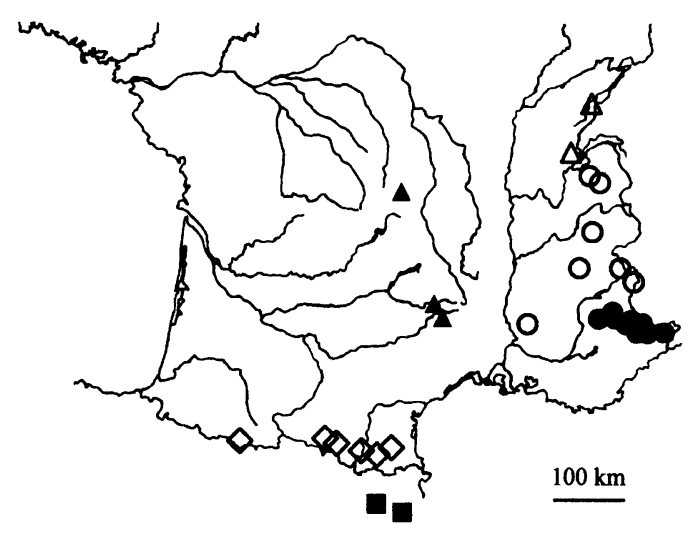

(b)

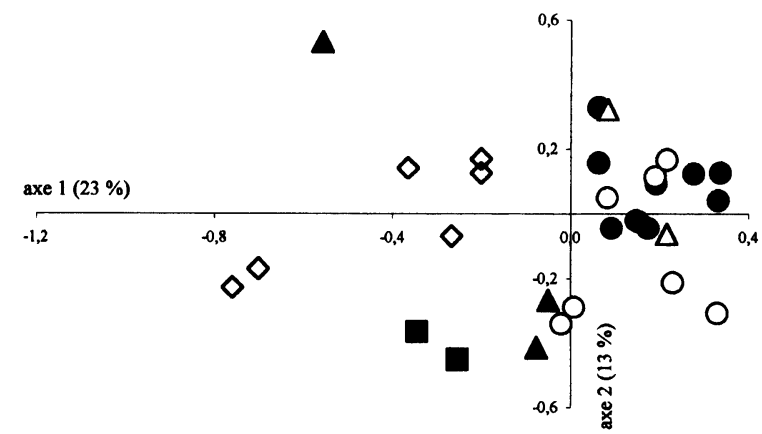

(c)

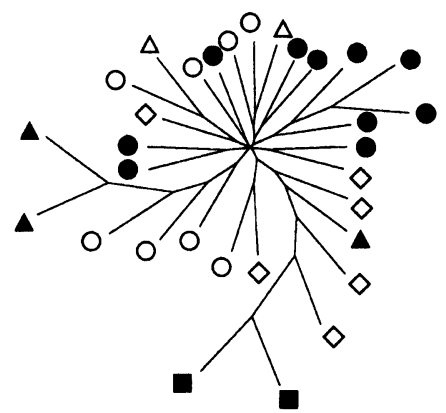

Figure 3. A. Position géographique des populations de $P$. apollo échantillonnées en France et en Espagne. Les populations sont représentées chacune par des signes différents selon les massifs montagneux et repris en $\mathrm{B}$ et $\mathrm{C}(\diamond$ : Pyrénées ; $\mathbf{\square}$ : Espagne ; $\boldsymbol{\Delta}$ : Massif Central ; $\triangle$ : Jura ; $\circ$ : Alpes au Nord des Alpes Maritimes ; $\bullet$ : Alpes Maritimes). B. AFC réalisée sur les fréquences alléliques aux loci analysés ( $c f$. Tab. I) ; pourcentage d'inertie représenté par le plan 1-2: $55 \%$. C. Phénogramme obtenu avec ces mêmes données et par la même méthode qu'en $1 \mathrm{C}$. 
exemple, dans le groupe alpin). À très grande échelle, la structure devient plus claire ; en particulier, les populations espagnoles sont très distinctes.

Pour l'ensemble à l'échantillonnage le plus serré, les Alpes-Maritimes, aucun effet d'isolement par la distance significatif n'est observé (Fig. 6C) ; mais en fait cela correspond à une valeur faible de $\theta$ ( 0.04 pour 10 populations) et il est vraisemblable que l'on se trouve à l'intérieur d'un même « voisinage ». Il ne faut pas oublier non plus que les vallées internes du massif des Alpes-Maritimes étaient couvertes de glaciers au Würm et que leur colonisation est récente. On retrouve un effet d'isolement par la distance au niveau de l'ensemble AlpesJura $\left(r^{2}=0.03\right.$, test de Mantel : $\left.p=0.006\right)$ et surtout de la totalité de la zone étudiée (Fig. 6D).

Un examen attentif des données apporte quelques déductions logiques. Très clairement, la diversité génétique est minimale dans le Massif Central et le Jura (et l'Espagne) et élevée dans les populations des Alpes et des Pyrénées (Tab. II). On a de plus relevé un déséquilibre gamétique significatif entre certains loci dans la population ariégeoise paradoxalement placée avec les alpines ; l'analyse de la contribution des variables aux axes de l'AFC montre que ce positionnement est dû à un faible nombre d'allèles (non figurés dans le présent travail) communs avec des populations alpines ; les critiques arguant des possibilités d'homoplasies dans les allozymes prennent ici tout leur poids. En revanche, le rattachement de la population de l'ouest du Massif Central aux Pyrénées semble significatif, comme nous le verrons plus loin.

L'interprétation des structures observées est évidemment délicate. Il faut faire intervenir non seulement les données contemporaines de la génétique des populations évoquées plus haut, mais aussi la biogéographie. Il est clair que l'Apollon a connu une histoire pléistocène mouvementée, qu'il est très difficile de reconstituer avec les faibles indices dont nous disposons. Au cours des phases les plus chaudes, les populations se sont réfugiées dans les massifs les plus élevés ou dans des poches précaires - phénomène qui est en train de se reproduire. Au plus dur des glaciations, elles se sont localisées dans le sud de l'Europe, à des altitudes inférieures de 1000 à $1200 \mathrm{~m}$ par rapport à leur position actuelle (soit entre le niveau de la mer et $1000 \mathrm{~m}$ ou un peu plus). L'emplacement des refuges est plus difficile à déterminer que dans le cas précédent ; il y a même pu y avoir à certains moments des peuplements subcontinus dans le sud de l'Europe, spécialement au cours des phases sèches, froides et ensoleillées, conditions très favorables à l'Apollon. Comme dans le cas de $P$. eunomia, le retrait des glaciers a entrainé une colonisation vers le haut et vers le nord ; il est clair que les régions situées au nord du $45^{\mathrm{e}}$ parallèle étaient inoccupées par l'espèce et ont subi une colonisation post-glaciaire. Il est vraisemblable en revanche que, par exemple, les colonies actuelles des environs du Ventoux ont pu pratiquement rester en place en changeant simplement d'altitude. Au contraire, des barrières efficaces à l'heure actuelle malgré leur altitude favorable, comme les monts de la Margeride, du Gévaudan et du Livradois, ont joué leur rôle en permanence ; le facteur semble être, tout simplement, l'absence de peuplements 
suffisants de plantes nourricières, liée à des facteurs édaphiques. En revanche, la variation géographique d'autres espèces de lépidoptères montagnards suggère des échanges importants entre les Pyrénées et l'Auvergne [32]. Les Apollons du Massif Central ne constitueraient donc pas un peuplement homogène : les populations de l'est seraient plus proches de celles des Alpes, et les populations de l'ouest de celles des Pyrénées

Le comportement migratoire de l'espèce, connue pour effectuer des fondations à longue distance (p. ex. [8]), est également à prendre en compte. Des déplacements individuels dépassant une dizaine de kilomètres, y compris à travers des zones défavorables, ont été observés [9] ; par ailleurs, les individus erratiques ont tendance à se déplacer tant qu'ils ne rencontrent pas une autre colonie (Descimon [12] et non publié). Dans ces conditions, la patterne de migration est susceptible d'être conforme à une distribution leptokurtique [30,55], avec beaucoup d'individus sédentaires et quelques autres migrant à longue distance. Les conséquences de ce comportement seraient à intégrer dans une variante du modèle d'isolement par la distance.

La dynamique très instable des populations et l'histoire mouvementée de l'espèce expliquent donc la complexité de la structure actuelle. Il est clair que des études beaucoup plus approfondies sont nécessaires à une meilleure compréhension de la structure génétique des populations de ce Parnassius, beaucoup plus complexe que celle de son congénère $P$. mnemosyne $[14,15]$. L'effort devra impliquer la recherche de nouveaux marqueurs génétiques et l'analyse fine des populations des diverses régions.

\subsection{Euphydryas aurinia, une espèce à distribution continue}

Cette Mélitée occupait dans un passé récent presque tout le territoire français, à l'exception du territoire des Pyrénées centrales et occidentales. Du niveau de la mer à la zone alpine des Alpes et des Pyrénées orientales, de la région méditerranéenne aux prés humides et aux tourbières du nord de la France, on trouvait des populations inféodées à des plantes nourricières variables. La destruction poussée des milieux naturels dans la moitié nord de la France a beaucoup amenuisé le peuplement de cette espèce, qui reste largement répandue dans la moitié sud. Une variation phénotypique importante, mais peu étudiée (à part un travail biométrique de Mazel [35]) suggère une très large variation génétique.

Nous avons réalisé une étude, utilisant le polymorphisme enzymatique, sur l'ensemble de la France, en tentant de recouvrir le territoire par un maillage large et en approfondissant l'étude dans certaines zones. Nous donnons ici un premier aperçu de ce travail.

Dans la moitié sud de la France, ce papillon fréquente une grande diversité de plantes nourricières [35]. Ces plantes appartiennent néanmoins, comme chez toutes les Mélitées, à un ensemble taxinomique défini, caractérisé par la 
(a)

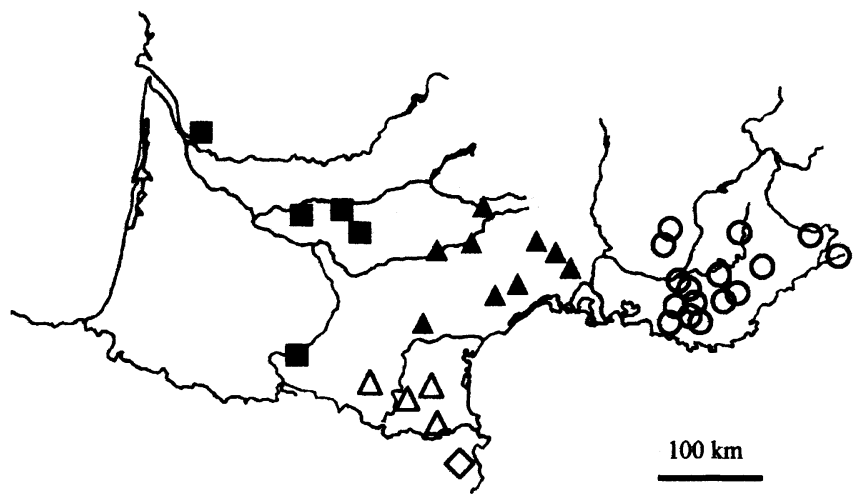

(b)

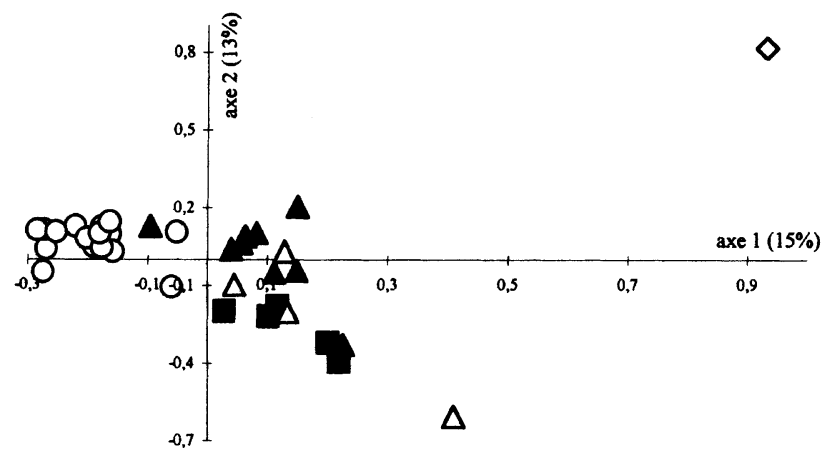

(c)

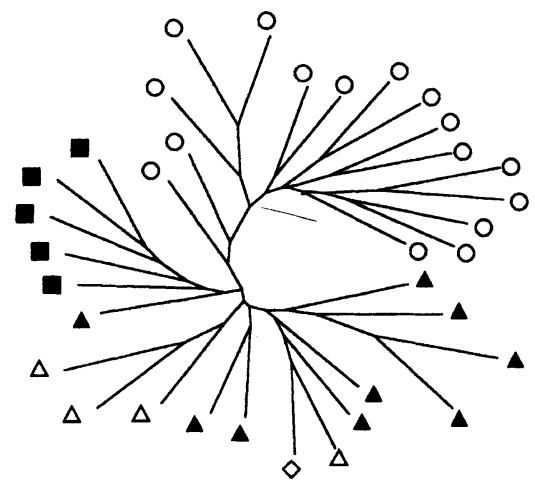

Figure 4. Position géographique de 35 populations d'E. aurinia analysées dans le Sud de la France et en Espagne ; ces populations sont affectées à 5 régions symbolisées chacune par un signe différent, repris en B et $\mathrm{C}$ ( 0 : Provence ; $\Delta$ : Languedoc ; $\diamond:$ Catalogne ; $\mathbf{\square}$ : sud-ouest ; $\triangle$ : Pyrénées orientales). B. AFC réalisée sur les fréquences alléliques aux loci analysés ( $c f$. Tab. I) ; pourcentage d'inertie représenté par le plan 1-2: $28 \%$. C. Phénogramme obtenu avec ces mêmes données et par la même méthode qu'en $1 \mathrm{C}$. 
présence de composés protecteurs, des glucosides iridoïdes [5]. Dans le cas présent, il s'agit de dipsacacées (Cephalaria, Knautia, Scabiosa, Succisa), de caprifoliacées (Lonicera), de valérianacées (Centranthus) et de gentianacées (Gentiana) [35]. Dans le nord, les populations sont liées pour l'essentiel Succisa pratensis et parfois à diverses espèces de Knautia.

Les travaux de Ehrlich et de son école, en Californie, ont montré que les échanges entre les colonies d'une autre espèce d'Euphydryas, E. editha, sont réduits - cette espèce a servi à définir l'opposition populations ferméespopulations ouvertes [16-18]. Qu'en est-il pour l'espèce européenne? De même, les modalités de son adaptation aux plantes nourricières sont-elles comparables à ce qui a été observé chez $E$. editha [45] ?

$E$. aurinia pose donc toute une série de questions : d'abord, par analogie avec $P$. eunomia et $P$. apollo, comment se présente la variation géographique de la structure génétique? L'inféodation à des plantes nourricières différentes est-elle le fait de souches différentes, ou bien cette caractéristique n'est-elle que superficielle, la structure génétique étant gouvernée par d'autres facteurs? Les échanges géniques sont-ils significatifs d'une structure ouverte ou fermée? Obéissent-ils à la règle de l'isolement par la distance ? Ceci a été l'objet d'un travail, encore non publié, effectué par deux d'entre nous (M. Zimmermann et B. Barascud), dont nous donnons ici un aperçu préliminaire.

Une étude effectuée d'abord sur les populations du Midi montre, par les deux méthodes utilisées ici (dendrogramme et plan 1-2 de l'AFC sur les fréquences alléliques) que la structuration est nette et clairement hiérarchisée en fonction de la localisation géographique (Fig. 4B, C). Les populations provençales forment un ensemble particulièrement cohérent, comme celles du Languedoc calcaire et celles du sud-ouest. Les « anomalies » sont en grande partie explicables, comme nous allons le voir, par une liaison avec les plantes nourricières. Celle-ci est extrêmement intéressante mais complexe. Le groupe provençal est pour l'essentiel lié à Cephalaria mais, dans la zone géographique occupée par ces populations, cette plante est la seule disponible régulièrement et en quantité. Parfois, au sein de zones à Cephalaria, on observe une population liée à des espèces des genres Scabiosa ou Knautia; elle ne se singularise pas au niveau des allozymes ; on peut aussi noter que les populations provençales consomment volontiers du Lonicera après l'hivernage, mais cette plante n'est pas choisie par les femelles (Singer, comm. pers.). La situation est identique pour les populations du Languedoc calcaire. En revanche, une population des monts du Sommail, en Languedoc cristallin, liée à Succisa pratensis, se regroupe clairement avec les populations du sud-ouest, qui consomment cette plante, et non avec celles inféodées à Cephalaria. Le cas des colonies des Pyrénées orientales et de Catalogne montre une sérieuse hétérogénéité du peuplement. Celles qui sont liées à Lonicera etrusca, se situent à part ; il s'agit de l'extrémité nord du groupe hispano-maghrébin, déjà bien distinct par la patterne alaire, et inféodé à cette plante. Mais bien plus frappant est le regroupement des deux autres populations de la même région qui vivent sur Succisa pratensis (Coustouges 
(a)

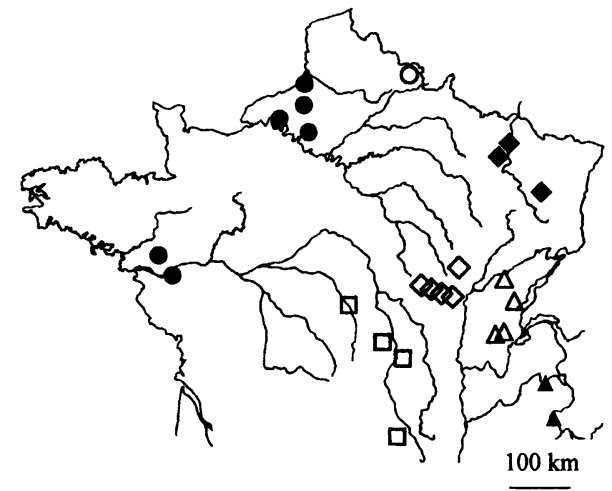

(b)

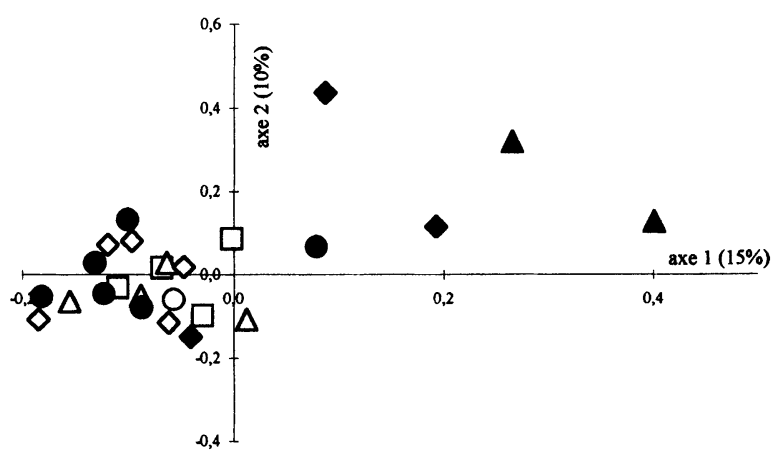

(c)

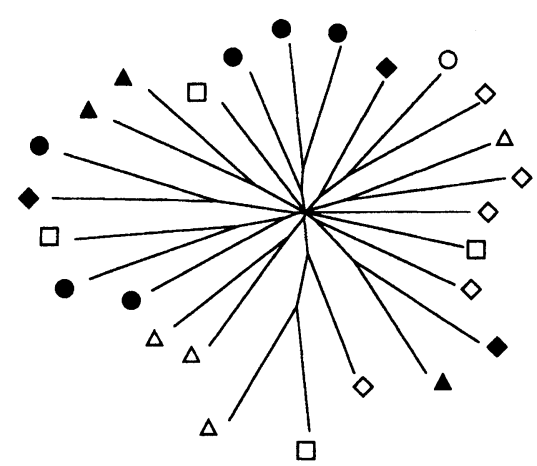

Figure 5. A. En haut, localisation géographique de 26 populations d'E. aurinia analysées dans la partie nord de la France; ces populations sont affectées à 8 régions symbolisées par un signe différent, repris en B et C $(\square$ : Centre de la France ; $\boldsymbol{\Lambda}$ : Alpes ; $\triangle$ : Jura $;$ : Lorraine ; $\circ:$ Nord ; $\bullet:$ Normandie et Ouest ; $\diamond:$ Bourgogne). B. AFC réalisée sur les fréquences alléliques aux loci analysés ( $c f$. Tab. I) ; pourcentage d'inertie représenté par le plan 1-2: $25 \%$. C. Phénogramme obtenu avec ces mêmes données et par la même méthode qu'en $1 \mathrm{C}$. 

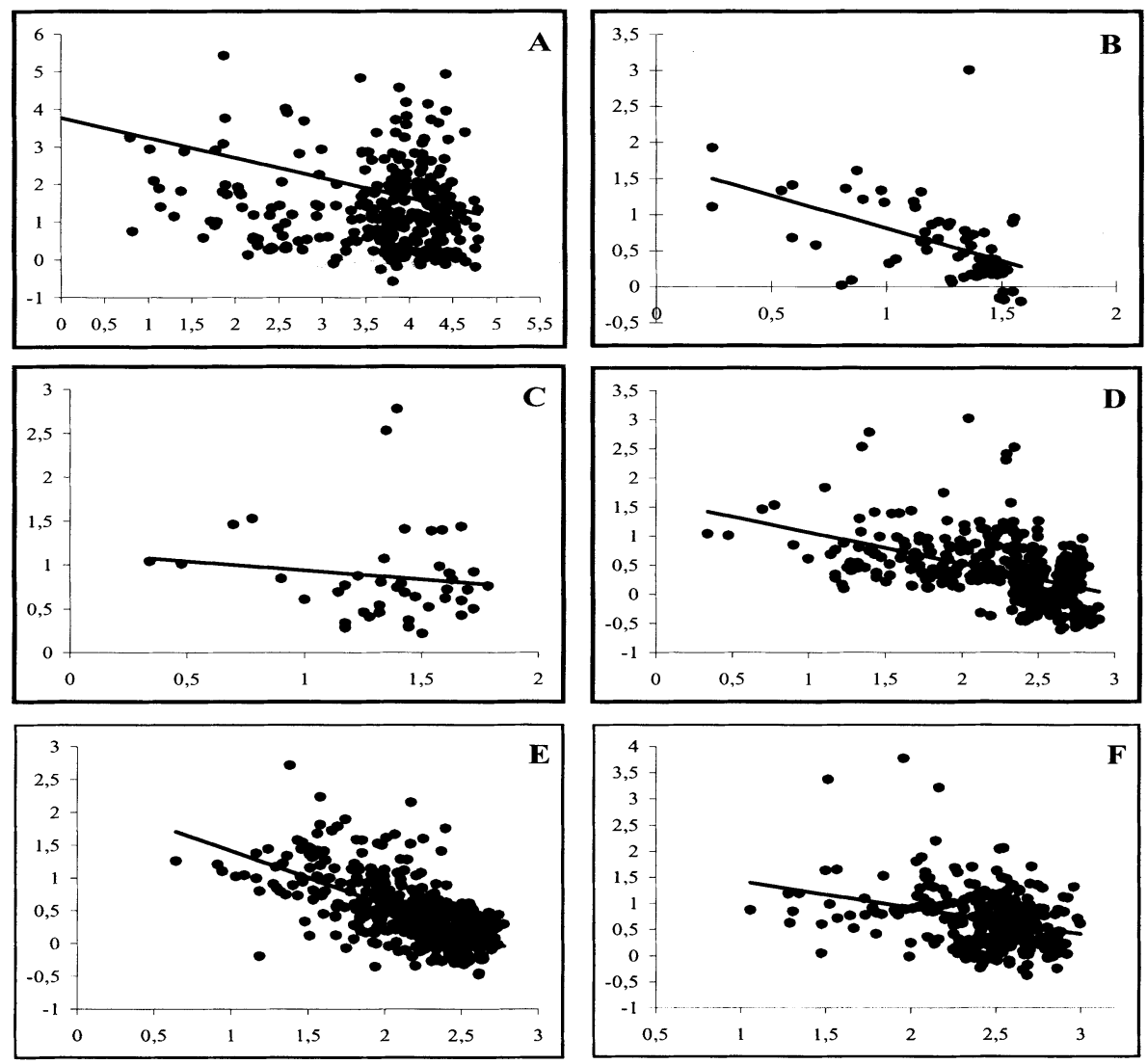

Figure 6. Diagrammes d'isolement par la distance selon Slatkin [46] : régression du logarithme de la distance géographique entre les populations (en abscisses) contre le logarithme du paramètre $N_{e} m$ (calculé à partir de $\theta$ ) (en ordonnées) chez 6 ensembles de populations des 3 espèces étudiées. Dans chaque cas, le nombre de populations concernées et les paramètres de la droite de régression et du coefficient de corrélation sont fournis. La significativité (probabilité d'écart par rapport à l'hypothèse nulle) est évaluée par le test de Mantel [31].

A. Populations de P. eunomia en Ardenne.

$N=26 ; y=-0,5333 x+3,7746 ; r^{2}=0,0632 ; P=0,012$.

B. Populations de $P$. eunomia dans les Pyrénées orientales.

$N=12 ; y=-0,9129 x+1,7239 ; r^{2}=0,261 ; P<0,001$.

C. Populations de $P$. apollo dans les Alpes maritimes.

$N=10 ; y=-0,208 x+1,1447 ; r^{2}=0,0166 ; P=0,288$.

D. Ensemble des populations analysées de $P$. apollo.

$N=31 ; y=-0,5399 x+1,6074 ; r^{2}=0,2296 ; P<0,0001$.

E. Populations d'E. aurinia du sud de la France.

$N=35 ; y=-0,8179 x+2,2310 ; r^{2}=0,4052 ; P<0,0001$.

F. Populations d'E. aurinia du nord de la France.

$N=26 ; y=-0,5047 x+1,9254 ; r^{2}=0,0939 ; P=0,004$. 
et Pailhères) avec le groupe du sud-ouest, lié à cette plante. Localisation géographique et spécialisation trophique influencent donc l'une et l'autre la structure génétique. En revanche, la liaison avec les espèces des genres Scabiosa et Knautia semble moins significative et se produit de manière inorganisée au milieu de groupes associés soit à Succisa pratensis, soit à Cephalaria Leucantha. L'analyse par la méthode de l'isolement par la distance (Fig. 6E) montre une corrélation très significative.

L'extension de l'analyse à l'ensemble du territoire français, gênée par les extinctions massives des colonies de la moitié nord-ouest, permet néanmoins de se rendre compte que la structuration est beaucoup moins nette et moins corrélée avec la géographie. Les populations se branchent confusément en un véritable buisson et l'AFC les regroupe en un paquet serré, s'opposant à la distribution étalée des populations du sud (Fig. 5B, C). De plus, aucune liaison nette n'est observée entre ces représentations et la localisation géographique, pourtant bien étalée. Il existe un effet d'isolement par la distance, mais moins net que dans le sud (Fig. 6F).

L'exemple de la Bourgogne, où il est possible de comparer des populations liées à Succisa pratensis et à une Knautia, montre qu'il n'apparaît pas, avec les moyens utilisés, une différenciation génétique associée à la plante nourricière. En fait, le peuplement de toute cette région correspond à celui localisé aux milieux frais du Midi. On notera qu'en Normandie, Succisa pratensis, et donc son phytophage, pousse sur des côteaux exposés au sud - un glissement écoclimatique assez classique.

Tout se passe comme si trois grands peuplements liés à des plantes et des milieux différents s'intriquaient : 1) un groupe méridional, atlanto-méditerranéen, lié à la forêt méditerranéenne thermophile et à une liane, le chèvrefeuille $L o$ nicera ; 2) un autre groupe, méridional et méditerranéen, mais vivant sur $C e ́-$ phalaria et lié à des milieux ouverts, plus froids ; 3) un groupe associé à des milieux plus atlantiques, frais et humides, limité aux zones montagnardes dans la région méditerranéenne, et associé à une plante de prés humides oligotrophes, succisa. Le passage sur des espèces des genres Knautia et Scabiosa semble être homoplasique. Nous noterons que les populations d'altitude liées à différentes espèces de gentianes Gentiania n'ont pas encore été étudiées en détail.

Il est clair que les populations méridionales sont génétiquement, comme dans le cas de $P$. eunomia, plus diverses et plus nettement structurées géographiquement (Tab. II) et dotées de potentialités adaptatives plus larges. La structure floue des populations nordiques a aussi la même signification : elle résulte d'une colonisation assez récente, postglaciaire. Un scénario biogéographique séduisant est que les trois grands peuplements de E. aurinia ont suivi les biomes auxquels ils sont adaptés dans les flux et reflux causés par les variations climatiques. Il nous semble vraisemblable que, dans le cas des régions méridionales, les refuges n'étaient pas situés très loin de l'emplacement actuel des peuplements. 


\section{DISCUSSION}

Les trois cas étudiés, malgré la grande différence de leurs caractéristiques écologiques et biogéographiques, montrent une convergence dans la structure génétique de leurs peuplements. Même pour les « reliques glaciaires », dont, par un contresens très répandu, il était admis qu'elles «venaient du nord », le maximum de variabilité est rencontré dans les populations méridionales. Cette convergence se retrouve à plusieurs niveaux. D'abord au niveau de la diversité génétique globale, mesurée par les divers estimateurs : pourcentage de loci polymorphes, nombre moyen d'allèles par locus, nombre d'allèles efficaces, taux d'hétérozygotie (Tab. II). À ce niveau, les trois espèces diffèrent quantitativement, mais l'étendue de la distribution et la diversité génétique globale au niveau de l'espèce varient dans le même sens. Les estimations de la diversité génétique doivent être interprétées avec précaution dans le cas des comparaisons interspécifiques ; dans le cas présent, la similitude des méthodes employées permet d'éviter un biais purement technique. Par ailleurs, bien évidemment, ce résultat nécessiterait d'être généralisé sur un échantillon spécifique plus nombreux. La tendance de la variation est en revanche identique chez les trois espèces : ce sont les peuplements méridionaux qui sont les plus divers, ce qui correspond très bien à ce qui a été mis en évidence chez d'autres organismes [28]. Les faits demandent néanmoins à être regardés de plus près, car ils ne sont pas déterminés simplement [22].

Le premier facteur à diminuer la diversité est le faible effectif efficace des populations, qui engendre de la dérive. Il peut être aggravé par l'isolement, qui réduit les échanges géniques. C'est ce qui a été observé, pour le peuplement du sud-est de la France de Parnassius mnemosyne [14] ; dans ce cas, il a pu être établi une corrélation très significative entre les estimations de la diversité génétique et un coefficient d'isolement, calculé à partir de l'aire des stations et de leur distance aux noyaux majeurs du peuplement (on pourrait même parler d'un «syndrôme d'isolement »). L'analyse est difficile à pratiquer sur des situations moins simples, et sans doute moins statiques, comme celles des trois espèces étudiées ici. Chez $P$. apollo, l'effet joue clairement ; en particulier, les populations du Massif Central, très fragmentées, montrent un « syndrôme d'isolement » net. Quant à E. aurinia, dont les populations étaient naguère assez continues, ce type d'approche perd de sa pertinence.

Chez les trois espèces, les estimateurs de la diversité génétique montrent régulièrement des valeurs plus élevées pour les peuplements méridionaux (même dans le cas moins typique de $P$. apollo, si on considère comme « nordiques » le Massif Central dans son ensemble et le Jura) ; le « nombre absolu de migrants $\gg\left(N_{e} m\right)$ y est aussi plus élevé (Tab. II). Cette diversification entre les populations n'est pas seulement plus élevée dans le Midi, elle est aussi plus structurée. Essentiellement géographique avec $P$. eunomia et, moins nettement, avec $P$. apollo, cette structuration met aussi en jeu, chez E. aurinia, la spécialisation trophique. Mais elle est en tout cas plus nette que dans le Nord. Remarquons qu'il ne s'agit pas du simple effet d'un moins grand nombre de 
loci polymorphes ; chez $P$. eunomia, si on ne garde dans une analyse des populations pyrénéennes que les loci polymorphes en Ardenne, on obtient encore des résultats clairs (Barascud, non publié).

Dans cette étude, les concepts de flux génique et d'isolement par la distance sont à manier avec précaution. Le calcul de $N_{e} m[54,55]$ est fondé sur un modèle extrêmement réducteur et reposant sur des hypothèses peu réalistes [53] ; les critiques de Bossart et Pashley Prowell [4] doivent aussi être prises en compte : en particulier, les zones d'intergradation entre des entités génétiques distinctes peuvent fausser les estimations de $N_{e} m$. Il est prudent de ne considérer comme significatifs que les tests effectués à l'intérieur d'unités cohérentes. Il est clair que, chez $P$. eunomia, les populations ardennaises et pyrénéennes n'échangent ni gènes ni individus à notre époque, alors qu'il est possible de calculer une valeur de $N_{e} m$. Il en est d'ailleurs de même pour les populations du Morvan et leur population mère ardennaise : le «nombre de migrants » qu'elles échangent est, selon les calculs de $\theta$, de 0.42 [1]. Or, nous savons qu'en réalité il y a eu effet des fondateurs en 1970 et 1973. Le modèle de Slatkin [46] prévoit la succession d'une phase de fondation et d'une phase de «lissage » par les flux géniques. Mais, dans un contexte de fonctionnement en métapopulation à turnover élevé, il n'y a pas de situation d'équilibre [21,27]. Les modalités $\mathrm{du}$ processus de colonisation peuvent elles aussi influencer profondément la structure des peuplements qu'il engendre [30].

Est-ce à dire que la méthode par régression n'a pas de signification ? Si on arrive à définir des « unités populationnelles » où un échange d'individus et de gènes est actuellement possible, la méthode doit pouvoir fonctionner, en combinaison avec les autres approches et, en particulier celles qui fournissent des représentations des structures et permettent de définir des groupes - les dendrogrammes et les AFC. Il ne faut pas oublier que l'approche prônée par les détracteurs des méthodes génétiques, l'observation directe, bute aussi sur un obstacle majeur : la difficulté d'observer et de quantifier les migrations et, surtout, d'en apprécier l'impact génétique réel. Dans ce cadre, la méthode de Slatkin reste instructive. Chez $P$. eunomia, le contraste entre la structure d'un massif méridional (Pyrénées orientales) avec l'Ardenne prend tout son sens. Dans le cas de $P$. apollo, la prédominance des effets stochastiques même à courte distance souligne que l'on n'a pas affaire à des populations en équilibre, mais à un ensemble de métapopulations où extinctions et recolonisations se succèdent à un rythme rapide.

En ce qui concerne E. aurinia, la grande étendue de la distribution et la diversité des habitats et des ressources donnent évidemment lieu à une situation riche et complexe. À l'intérieur d'ensembles homogènes, comme le peuplement provençal, par exemple, une corrélation largement significative entre distance et $N_{e} m$ suggère que le modèle de l'isolement par la distance s'applique au moins en partie à l'intérieur de la région. Avec les outils dont nous disposons, il est difficile de dire si les affinités génétiques entre les populations utilisant la même plante nourricière résultent de leur appartenance à une souche colonisatrice 
adaptée à celle-ci ou bien à des pressions sélectives similaires sur les génotypes analysés. Dans ce dernier cas, les ressemblances génétiques seraient dues à des convergences ; des travaux récents [19] incitent à prendre au sérieux cette hypothèse, naguère rejetée dans le cadre de la théorie neutraliste. Dans la moitié nord de la France, le problème se pose beaucoup moins, Succisa pratensis étant pratiquement la seule plante nourricière. En revanche, contrairement à la moitié sud, la structure génétique n'est pas très ordonnée et l'effet de l'isolement par la distance y assez faible. Cette opposition est très parallèle à celle qui a été observée par Britten et al. [7] chez les populations d'Euphydryas editha dans le Grand Bassin et les Montagnes Rocheuses Centrales aux États-Unis.

Dans le « triangle »formé par les trois espèces, il y a finalement plus de ressemblance dans la variation de la structure génétique entre $P$. eunomia et $E$. aurinia qu'entre celles-ci et $P$. apollo. On notera simplement que la taille et la puissance de vol des deux nymphalides est inférieure, et aussi qu'elles consomment beaucoup moins de ressources alimentaires par individu que le papilionide. Cette remarque souligne l'importance d'une coordination entre des recherches génétiques et écologiques.

\section{CONCLUSION}

Du côté des outils, le présent travail n'avait pas la prétention d'effectuer un test absolu. Les enzymes, malgré leurs faiblesses criantes (résolution médiocre, homoplasies par mutations homologues, impossibilité de rejeter ou de démontrer l'action de forces sélectives...), permettent toujours de mettre en évidence des structures de populations. Il est clair que ce qui est trouvé correspond à une partie au moins de la réalité génétique, comme le montre le parallélisme entre les résultats de l'analyse du polymorphisme enzymatique et celle de la patterne alaire. Bien entendu, dans certains cas, des discordances ont été révélées entre les informations apportées par les allozymes et celles provenant d'autres marqueurs [4]. Par ailleurs, s'il est permis d'espérer qu'au prix d'un effort plus important que pour d'autres groupes, même voisins (hyménoptères, pucerons par exemple), des marqueurs microsatellites viendront épauler les enzymes, il ne paraît pas raisonnable d'en attendre trop [38]. En fait, il semble que la vraie nouveauté viendrait d'un approfondissement de l'approche sur les enzymes, au niveau du gène et non plus de la protéine. La connaissance des séquences permettrait entre autres de révéler beaucoup plus de variabilité, de détecter des homoplasies par « allèles isoactifs » [47], de disposer d'informations sur les événements sélectifs qui ont pu affecter les loci dans les différents haplotypes [3] et de les replacer au sein des blocs chromosomiques. Cette approche est en cours en ce qui concerne les enzymes de la glycolyse et la puissance de vol chez les Colias [50,51] ; elle pourrait s'étendre à d'autres aspects de l'adaptation - dont celle aux plantes nourricières. 
Du côté des méthodes d'analyse, il est apparu clairement que les diverses méthodes de représentation des relations génétiques entre les populations sont tout simplement complémentaires et qu'il est inutile de les opposer. Le paramètre $N_{e} m$ n'est au fond qu'une distance génétique qui a le mérite d'être assez parlante. Les analyses en espaces réduits, en particulier dans leurs développements les plus récents, semblent ne pas avoir révélé toutes leurs possibilités dans le domaine de la distribution spatiale de la variabilité et de l'analyse du déséquilibre de liaison entre loci multiples. De même, l'étude de la variation spatiale de la structure génétique doit être effectuée de manière intégrée, sous peine de tomber dans les pièges dénoncés par Bossart et Pashley Prowell [4], en particulier de prendre en compte des zones hybrides entre des ensembles populationnels différents. Nous pensons d'ailleurs que les paramètres tirés de l'analyse - significativité de la corrélation, pente de la droite de régression, ordonnée à l'origine - gardent une signification même si le modèle schématique de l'isolement par la distance n'est pas respecté.

Les résultats proprement dits soulignent l'influence de la géographie européenne et de l'histoire quaternaire des peuplements dans la structure génétique de ceux-ci. Pour deux des espèces, $P$. eunomia et $E$. aurinia, le contraste est vif entre les populations du sud de l'Europe, diverses et différenciées, à la structuration nette, et les populations du nord, récemment arrivées, où la variation interpopulations est désordonnée et la diversité intrapopulation faible. Cette différence peut être attribuée, pour une part au moins, à la colonisation récente, postglaciaire, des régions septentrionales, accompagnée d'effet des fondateurs, de dérive et de balayages sélectifs. Les colonies méridionales sont presque restées sur place, en se contentant de remonter avec les étages de végétation dans les refuges montagneux, et n'ont donc pas subi les goulots d'étranglement liés à une dispersion par bonds. Il ne faut néanmoins pas oublier que l'Europe du sud présente une topographie et un climat et donc des milieux et des ressources bien plus diversifiés que l'Europe du nord. La variété des plantes nourricières utilisées par E. aurinia dans le Sud est là pour le rappeler, mais l'argument n'est pas valable pour $P$. eunomia, monophage. $P$. Apollo ne présente pas une tendance aussi claire ; ici, ce sont simplement les petites populations des massifs de basse et moyenne montagne qui sont moins diverses génétiquement par rapport à celles des massifs alpin et pyrénéen. Les colonies les plus septentrionales en France (Vosges, nord du Jura) sont éteintes et celles de l'Eifel en Allemagne ne nous ont pas été accessibles.

Si nos résultats s'intègrent bien dans la vision générale de la biogéographie de l'Europe occidentale proposée par Hewitt [28], ils montrent aussi qu'il est dangereux de généraliser l'hypothèse selon laquelle, pendant les glaciations, toute la zone comprise entre le front glaciaire du nord de l'Europe et les refuges de l'extrême sud du continent était une toundra absolument déserte. C'est certainement vrai pour les espèces thermophiles. Mais, à l'heure actuelle, $P$. apollo et $E$. aurinia peuvent vivre dans nos régions à plus de 2000 mètres d'altitude, en milieu très ouvert et sous des conditions très rudes ; $P$. eunomia se trouve 
en Scandinavie jusqu'à la limite Nord de la forêt boréale. Il serait surprenant que le Sud de la France leur ait été totalement inhospitalier.

$\mathrm{Au}$ point de vue de la conservation de la diversité génétique, une caractéristique apparemment favorable est que cette dernière est concentrée dans le Sud, où les milieux sont moins dégradés et les populations moins fragmentées. Les espaces protégés (parcs nationaux et régionaux, réserves) y sont aussi plus nombreux. Mais une menace sévère pèse sur cette situation : le réchauffement global provoque le glissement vers le nord des aires des espèces [43] ; les colonies les plus méridionales de certaines espèces disparaissent, alors qu'il y a extension à la limite nord. Au niveau de la diversité génétique, il n'y a pas symétrie, puisque, si ce que nous avons observé est général, ce qui est perdu était riche et ce qui s'étend est pauvre. Comme bien d'autres, le présent travail souligne donc la gravité des conséquences biologiques du changement climatique rapide découlant des activités humaines et de la nécessité de le modérer et de le ralentir.

\section{REMERCIEMENTS}

Nous remercions toute l'équipe du Bureau des ressources Génétiques pour l'aide qui a été apportée à notre laboratoire depuis de nombreuses années. L'auteur senior exprime une reconnaissance particulièrement vive à Louis Thaler, qui a su l'encourager au long d'un démarrage difficile, et à Yvette Dattée, qui a aussi montré beaucoup de bienveillance pour notre travail. Les Parcs Nationaux des Cévennes, du Mercantour et des Écrins ont facilité les activités de certains de nos collaborateurs, ainsi que les Parcs Naturels Régionaux du Haut-Jura et des Volcans d'Auvergne. Les autorisations d'étude, de capture et d'élevage des espèces considérées ont été accordées par le ministère de l'Environnement, qui a également subventionné une partie des études menées sur les Parnassius, P. eunomia et les mélitées. Nous tenons également à remercier l'Office pour l'information éco-entomologique (OPIE), et en particulier Solange et Robert Guilbot qui se sont chargés de certaines démarches administratives et ont fourni des financements de dépannage.

\section{RÉFÉRENCES}

[1] Barascud B., Contribution à l'étude génétique d'une espèce dont l'habitat est fragmenté à différentes échelles : Proclossiana eunomia (Esper, 1799) (Lepidoptera, Nymphalidae), Thèse, Université de Provence, (1996).

[2] Barascud B., Martin J.-F., Baguette M., Descimon H., Genetic consequences of an introduction-colonization process in an endangered butterfly species, J. Evol. Biol. 12 (1999) 697-709. 
[3] Begun D.J., Aquadro C.F., Levels of naturally occuring DNA polymorphism correlate with recombination rates in D. melanogaster, Nature 356 (1992) 51920.

[4] Bossart J.L., Pashley Prowell D., Genetic estimates of population structure and gene flow: limitations, lessons and new directions. Trends Ecol. Evol. 13 (1998) 202-206.

[5] Bowers D.M., Chemistry and coevolution : iridoids glycoside, plants, and herbivorous insects, in : K. Spencer (ed.), Chemical mediation of coevolution, Academic Press, New York, 1988, pp. 133-165.

[6] Braconnot S., Structure génétique de Parnassius apollo Linné (Lepidoptera Papilionidae) en France : étude du polymorphisme enzymatique, Thèse, Université de Provence, (1997).

[7] Britten H.B., Brussard P.F., Murphy D.D., Ehrlich P.R., A test for isolationby-distance in central Rocky Mountain and Great Basin populations of Edith's checkerspot butterfly (Euphydryas editha) J. Hered. 86 (1995) 204-210.

[8] Capdeville P., Les races géographiques de Parnassius apollo, Éditions Sciences Nat, Venette, 1978.

[9] Cosson E., Étude sur la biologie, l'écologie et la répartition dans le Jura de Parnassius apollo L. (Lepidoptera: Papilionidae), Mémoire de D.E.A., Université de Provence, 1995.

[10] Deschamps-Cottin M., Roux M., Descimon H., Valeur trophique des plantes nourricières et préférence de ponte chez Parnassius apollo L. (Lepidoptera, Papilionidae), C.R. Acad. Sci. Paris 320 (1997) 399-406.

[11] Descimon H., La pigmentation des lépidoptères: un modèle pour la théorie synthétique de l'évolution, Bull. Soc. Zool. Fr. 108 (1983) 303-312.

[12] Descimon H., La conservation des Parnassius en France: aspects zoogéographiques, écologiques, démographiques et génétiques, Insectes (Suppl.), 1994.

[13] Descimon H., Vesco J.P., A mutant affecting wing pattern in Parnassius apollo (Linné), J. Res. Lepidoptera 26 (1988) 161-72.

[14] Descimon H., Napolitano M., Enzyme polymorphism, wing pattern variability and geographical isolation in an endangered butterfly species, Biol. Cons. 66 (1993) 117-23.

[15] Descimon H., Napolitano M., Genetic structure of French populations of the mountain butterfly Parnassius mnemosyne L. (Lepidoptera : Papilionidae), Biol. J. Linn. Soc. 53 (1994) 325-41.

[16] Ehrlich P., Ehrlich, P.R., Intrinsic barriers to dispersal in Checkerspot butterfly. Science 134 (1961) 108-109.

[17] Ehrlich P.R., Raven P.H., The differentiation of populations, Science 165 (1969) 1128-32.

[18] Ehrlich, P.R., The structure and dynamics of butterfly populations, In : VaneWright, R.I., Ackery, P.R. (eds.), The Biology of Butterflies, Symposium 11 of the Royal Entomological Society, Academic Press, London, 1984, pp. 25-40.

[19] Feder L.D., Berlocher S.H., Opp S.B., Sympatric host-race formation and speciation in Rhagoletis (Diptera: Tephritidae): a tale of two species for Charles D., in : Mopper S. and Stauss S.Y. (eds), Genetic structure and local adaptation in natural populations, Chapman and Hall, London and New York, 1997, pp. 408-441.

[20] Ford E.B., Butterflies, Collins, London, 1945. 
[21] Gilpin M., The genetic effective size of a metapopulation, Biol. J. Linn. Soc. 42 (1991) 165-175.

[22] Godelle B., Austerlitz F., Brachet S., Colas B., Cuguen J., Gandon S., Gouyon P.H., Lefranc M., Olivieri I., Reboud X., Vitalis R., Système génétique, polymorphisme neutre et sélectionné : implications en biologie de la conservation, Genet. Select. Evol. 30 (Suppl. 1) (1998), S15-S28.

[23] Guinand B., Use of a multivariate model using allele frequency distributions to analyse patterns of genetic differentiation among populations, Biol. J. Linn. Soc. 58 (1996) 173-195.

[24] Hanski H., Metapopulation Ecology, Oxford University Press, Oxford, 1999.

[25] Hanski I., Kuussaari M., Nieminen, M., Metapopulation structure and migration in the butterfly Melitaea cinxia. Ecology 75 (1994) 747-762.

[26] Hanski I., Pakkala T., Kuussaari M., Lei, G.C., Metapopulation persistence of an endangered butterfly in a fragmented landscape, Oikos 72 (1995) 21-28.

[27] Harrison S., Local extinction in a metapopulation context: an empirical evaluation, Biol. J. Linn. Soc. 42 (1991) 73-88.

[28] Hewitt G.M., Post-glacial recolonization of European biota, Biol. J. Linn. Soc. 68 (1999) 87-112.

[29] Hillis D.M., Moritz C., Mable B.K., Molecular systematics, 2nd edition, Sinauer, Sunderland, Mass., 1996.

[30] Ibrahim K., Nichols R.A. Hewitt G.A., Spatial patterns of genetic variation generated by different forms of dispersal during range expansion, Heredity 77 (1996) 282-291.

[31] Legendre P., Legendre L., Numerical Ecology, Elsevier, Amsterdam, 1998.

[32] Lesse H. de, 1951, Etude biométrique des formes d'Erebia epiphron (Knoch), des Vosges, d'Auvergne et des Pyrénées, Rev. Fr. Lépidoptérologie 13 (1951) 3-9.

[33] Levins R., Extinction, in : Gerstenhaber, M. (ed.), Some Mathematical Questions in Biology. Lectures on Mathematics in the Life Sciences. Vol. 2., American Mathematical Society, Providence (R.I.), 1970, pp. 77-107 .

[34] Lewontin R.C., The Genetic Basis of Evolutionary Change, Columbia University Press, New York, 1974).

[35] Mazel R., Structure et évolution du peuplement d'Euphydryas aurinia Rottemburg (Lepidoptera) dans le sud-ouest européen, Vie Milieu 36 (1986), 205-225.

[36] Meglécz E., Solignac M., Microsatellite loci for Parnassius mnemosyne (Lepidoptera), Hereditas 128 (1998) 179-180.

[37] Nève G., Dispersion chez une espèce à habitat fragmenté : Proclossiana eunomia (Lepidoptera, Nymphalidae), Dissertation doctorale, Université catholique de Louvain, 1996.

[38] Nève G., Meglécz E., Microsatellite frequencies in different taxa, Trends Ecol. Evol. 15 (2000) 376-377.

[39] Nève G., Barascud B., Windig J.J., Population biology of Proclossiana eunomia (Nymphalidae): Preliminary results on morphometric and allozyme variation in Belgian and French populations, Nota Lepidopterologica, suppl. 5 (1994) 3-12.

[40] Nève G., Barascud B., Hughes R., Baguette M., Aubert J., Descimon H., Lebrun P., Dispersal, colonisation power and metapopulation structure in the vulnerable butterfly Proclossiana eunomia (Lepidoptera, Nymphalidae). J. Appl. Ecol. 33 (1996) 14-22. 
[41] Nève G., Mousson L., Baguette M., Adult dispersal and genetic structure of butterfly populations in a fragmented landscape. Acta œecol. 17 (1996) 621-626.

[42] Nève G., Barascud B., Descimon H., Baguette M., Genetic structure of Proclossiana eunomia populations at the regional scale (Lepidoptera, Nymphalidae), Heredity 84 (2000) 657-666.

[43] Parmesan C., Ryrholm N., Stefanescu C., Hill J.K, Thomas C.D., Descimon H., Huntley B., Kaila L., Kullberg J., Tammaru T., Tennent J., Thomas J.A., Warren, M., Poleward shifts in geographical ranges of butterfly species associated with regional warming, Nature 399 (1999) 579-83.

[44] Raymond M., Rousset F., GENEPOP (ver. 1.2), a population genetics software for exact tests and ecumenicism, J. Hered. 86 (1995) 248-249.

[45] Singer M.C., Thomas C.D., Evolutionary responses of a butterfly metapopulation to human- and climate-caused environmental variation, Am. Nat. 148 (supplement) (1996) 9-39.

[46] Slatkin M., Isolation by distance in equilibrium and non-equilibrium populations. Evolution 47 (1993) 264-279.

[47] Solignac M., Périquet G., Anxolabéhère D., Petit C., Génétique et évolution, tome I, Hermann, Paris, 1995.

[48] Sokal R.R., Oden N.L., Spatial autocorrelation analysis as an inferential tool in population genetics. Am. Nat. 138 (1991) 518-521.

[49] Swofford D.L., Selander R.B., Biosys-1 : a Fortran program for the comprehensive analysis of electrophoretic data in population genetics and systematics, J. Hered. 72 (1981) 281-283.

[50] Watt W.B., Cassin R.C., Swan M.S., Adaptation at specific loci. III. Field behaviour and survivorship differences among Colias PGI genotypes are predictable from in vitro biochemistry, Genetics 103 (1983) 725-739.

[51] Watt W.B., Biochemistry, physiological ecology, and population genetics - the mechanistic tools of evolutionary biology, Funct. Ecol., 5 (1991) 145-154.

[52] Weir B.S., Cockerham C.C., Estimating F-statistics for the analysis of population structure, Evolution 38 (1984) 1358-1370.

[53] Whitlock, M.C., McCauley, D.E., Indirect measures of gene flow and migration : $F_{S T} \neq 1 /(4 N m+1)$, Heredity 82 (1999) $117-125$.

[54] Wright S., Isolation by distance, Genetics 16 (1943) 114-138.

[55] Wright S., The genetical structure of populations, Ann. Eugen. 15 (1951) 323-54.

[56] Wright S., Evolution and the Genetics of Populations, Vol. 4, Variability within and among Natural Populations, University of Chicago Press, Chicago, 1978.

[57] Wynne I.R., Loxdale H.D., Brookes C.P. Use of cellulose acetate system for allozyme electrophoresis, in : Berry R.J., Bradshaw A.D., Crowford T. (eds.), Genes in Ecology, Blackwell, Oxford, 1992, pp. 494-499. 